\title{
Magnetic Assembly of Nanocubes for Orientation-Dependent Photonic Responses
}

Zhiwei Li, Mingsheng Wang, Xiaoliang Zhang, Dawei Wang, Wenjing Xu, and Yadong Yin* Department of Chemistry, University of California, Riverside, CA 92521 (USA).

*E-mail: yadong.yin@ucr.edu

\section{Chemicals}

Potassium ferricyanide, $\mathrm{HCl}$ and $\mathrm{NH}_{4} \mathrm{OH}$ were purchased from Fisher Scientific. Tetraethylorthosilicate (TEOS, 98\%), diethylene glycol (DEG, reagent grade), ethyl alcohol (EG, denatured), polyvinylpyrrolidone (PVP, Mw=40000) and 2-Hydroxy-2-methylpropiophenone (97\%) as photoinitiator were purchased from Sigma-Aldrich. Acrylamide (AM) and N, N'Methylenebisacrylamide (BIS) were obtained from Fluka.

\section{Synthesis of magnetic nanocubes}

Synthesis of PB nanocubes. PB nanocubes with an edge length of about $200 \mathrm{~nm}$ were synthesized according to a reported method. $113.14 \mathrm{mg}$ potassium ferricyanide and $3 \mathrm{~g}$ PVP $(\mathrm{Mw} .=40000)$ were added into $40 \mathrm{~mL}$ of $0.01 \mathrm{M} \mathrm{HCl}$. The solution was magnetically stirred for 30 min and then heated at $80^{\circ} \mathrm{C}$ for 20 hours. The obtained PB nanocubes were dispersed in 6 mL DI water.

$\mathrm{SiO}_{2}$ coating. $3 \mathrm{~mL}$ of the above colloidal dispersion of $\mathrm{PB}$ was added to the mixture of $10 \mathrm{~mL}$ of DI water and $70 \mathrm{~mL}$ of ethanol, which was then sonicated for $\sim 10$ min for better dispersion. Consequently, $3 \mathrm{~mL}$ of ammonia and $55 \mu \mathrm{L}$ of TEOS were added. The mixture was magnetically stirred overnight. The obtained $\mathrm{PB} @ \mathrm{SiO}_{2}$ was washed by DI water three times and finally concentrated in $\sim 50 \mu \mathrm{L}$ of DI water for following polyol reduction.

Polyol reduction. After silica coating, the $\mathrm{PB} @ \mathrm{SiO}_{2}$ was concentrated into about $50 \mu \mathrm{L}$ in DI water, which was then injected into $25 \mathrm{~mL}$ of $\mathrm{DEG}$ at $300{ }^{\circ} \mathrm{C}$ under nitrogen protection. Reduction typically took 12 hours under continuous nitrogen flow. Afterward, the products were washed by DI water three times. 


\section{Magnetic assembly of nanocubes}

$\mathrm{Fe}_{3} \mathrm{O}_{4} @ \mathrm{SiO}_{2}$ nanocubes were dispersed in $3 \mathrm{~mL}$ of ethanol with the concentration of $\sim 20 \mathrm{mg} / \mathrm{mL}$. The dispersion was then exposed to a magnet $(2000 \mathrm{G})$ for 20 seconds. After removing the magnet, $\mathrm{NH}_{4} \mathrm{OH}(20 \mu \mathrm{L})$ and TEOS $(6 \mu \mathrm{L})$ were added sequentially. The dispersion was then vortexed gently for 30 minutes. The obtained nanochains were washed by ethanol three times.

\section{Characterization}

TEM images were taken on a Tecnai T12 transmission electron microscope operating at $120 \mathrm{kV}$. The magnetic properties of cubic $\mathrm{Fe}_{3} \mathrm{O}_{4} @ \mathrm{SiO}_{2}$ were measured using a Lakeshore vibrating sample magnetometer equipped with 736 VSM controller, Model 142A, Model 642 Electromagnet power supply, and Model EM4 HV electromagnet. Optical diffraction spectra were measured by the Ocean Optics HR2000CG-UV-NIR spectrometer with a six-around-one reflection/backscattering probe. The angle between the incident light and the surface of the capillary was set at $30^{\circ}$. A home-made goniometer was used to precisely measure the angles between reflected light and the capillary surface. Dark-filed optical microscopic images were acquired by using A Zeiss AXIO Imager optical microscope. An X-ray diffractometer (XRD, EMPYREAM, PANalytical, $\mathrm{Cu}-\mathrm{K} \alpha$ radiation) was used to characterize the crystals phases of nanocubes after reduction. Dynamic light scattering (DLS) measurement was conducted in Delsa NanoC Particle Analyzer from Beckman Coulter.

\section{Simulation of magnetic field distribution}

The magnetic field distribution was simulated by a finite-element method performed on COMSOL Multiphysics. Background magnetic field was set parallel to the $\mathrm{x}$-axis with the filed strength of $1 \mathrm{mT}$. Three typical assembly modes were modulated as [100], [110], and [111]. The 
magnetization of nanocubes was set as $12006 \mathrm{~A} / \mathrm{m}$ at the applied field strength of $1 \mathrm{mT}$ based on the magnetization curve in Figure 1d. The magnetic susceptibility of surroundings was 1.

\section{Simulation of electric field distribution}

The electric field distribution was simulated by a finite-element method performed on COMSOL Multiphysics as well. The $\mathrm{Fe}_{3} \mathrm{O}_{4} @ \mathrm{SiO}_{2}$ nanocubes were modulated with a length of $250 \mathrm{~nm}$. The cross-section of a nanochain with 27 nanocubes aligned along their [110] direction inside was

constructed (Figure S7). The average refractive indices were 2.2 for domains of nanocubes and 1.333 for domains of water. A Gauss beam, S-polarized, was incident from the top with the predesigned wavelength (wl), spot radius (w0) of $12000 \mathrm{~nm}$. Its Rayleigh range was dependent on the incident wavelength with the formula of $\pi^{*} \mathrm{w} 0^{\wedge} 2 / \mathrm{wl}$.

\section{Printing color by lithography}

$\mathrm{Fe}_{3} \mathrm{O}_{4} @ \mathrm{SiO}_{2}$ nanocubes were dispersed in hydrogel precursor containing AM (250 mg), BIS (14 $\mathrm{mg})$, photoinitiator $(3 \mu \mathrm{L})$ in $\mathrm{EG}(1 \mathrm{~mL})$, which was then loaded between a glass substrate and a coverslip. A pre-designed photomask was placed above the devices, and then permanent magnet was applied parallelly to the surface of the cover glass. The polymerization was initiated by UV lamp with a wavelength of $254 \mathrm{~nm}$ and took 90 seconds totally. 

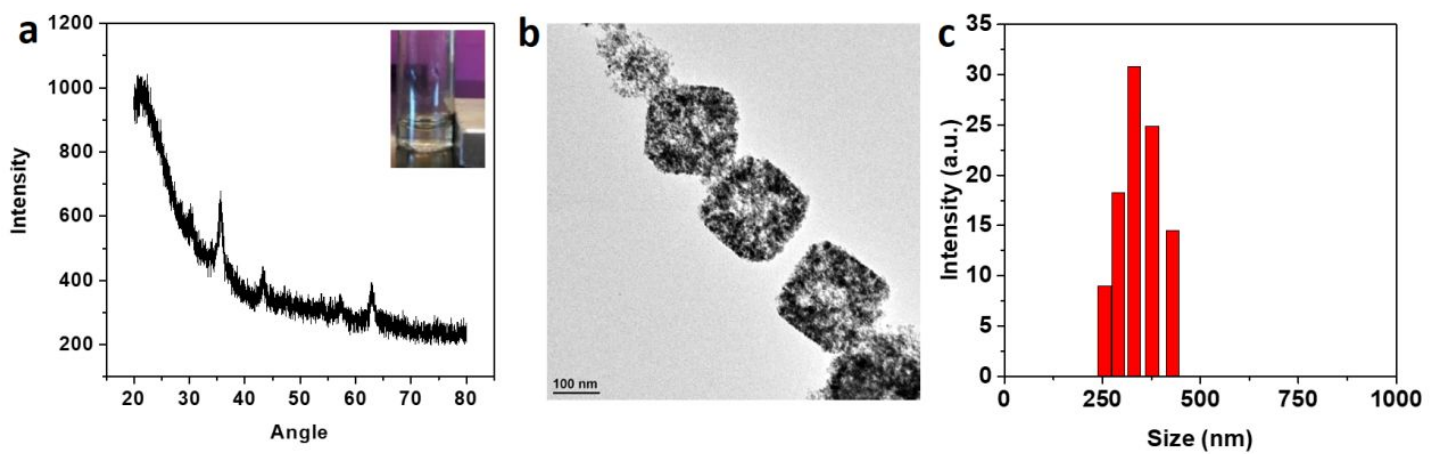

Figure S1. (a) $\mathrm{XRD}$ spectra of $\mathrm{Fe}_{3} \mathrm{O}_{4} @ \mathrm{SiO}_{2}$ nanocubes. Insert shows that $\mathrm{Fe}_{3} \mathrm{O}_{4} @ \mathrm{SiO}_{2}$ nanocubes can be magnetically separated from the colloidal solution. (b) TEM image of the corresponding sample after etching away the silica layer. (c) Size distribution of the $\mathrm{Fe}_{3} \mathrm{O}_{4} @ \mathrm{SiO}_{2}$ nanocubes measured by dynamic light scattering. 

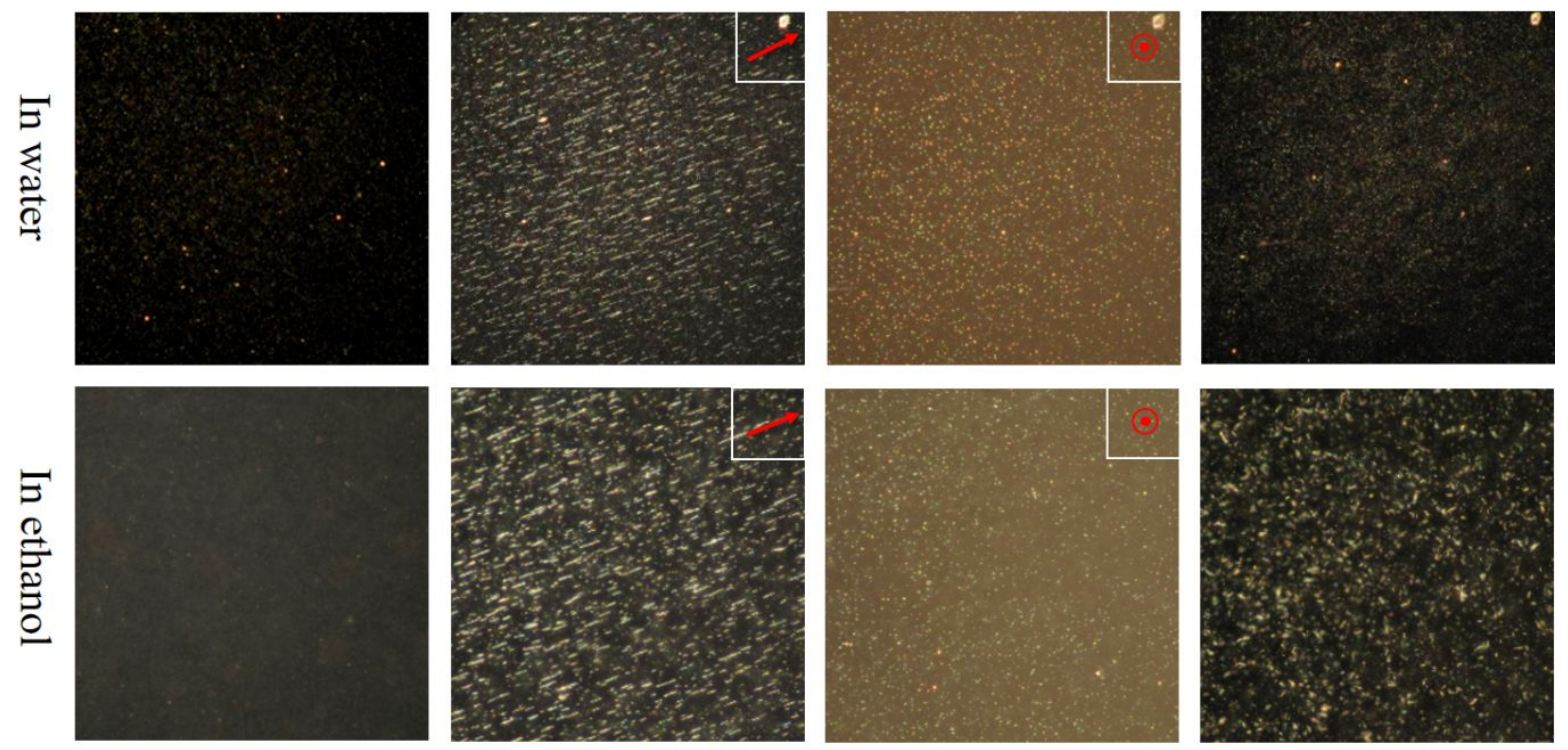

Without magnet With magnet (parallel) With magnet (vertical) Removing magnet

Figure S2. Dark-field optical microscopic images of a colloidal dispersion of $\mathrm{Fe}_{3} \mathrm{O}_{4} @ \mathrm{SiO}_{2}$ in pure water and ethanol. Red arrows indicate the direction of the external magnetic field. 

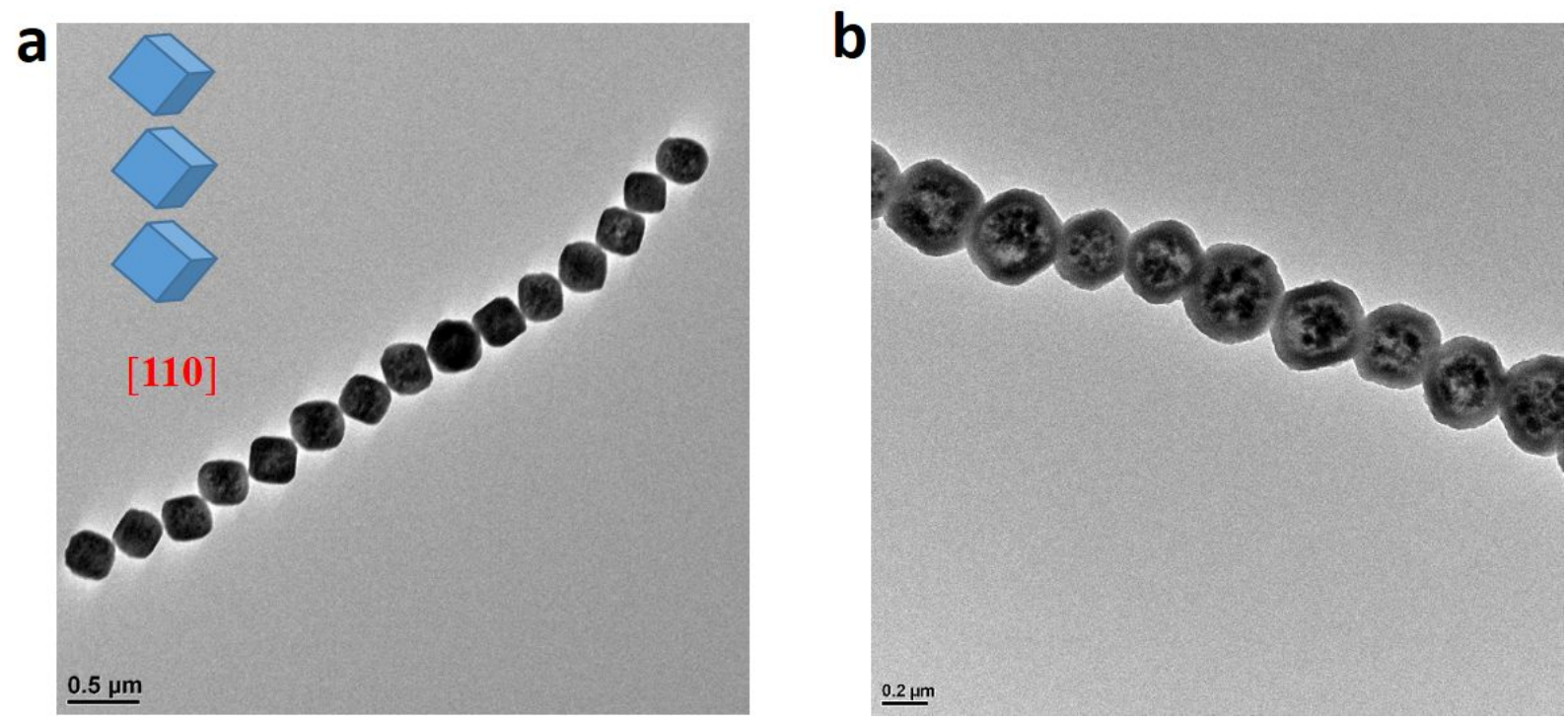

Figure S3. (a) and (b) TEM images of assembled chains showing orientational defects. 

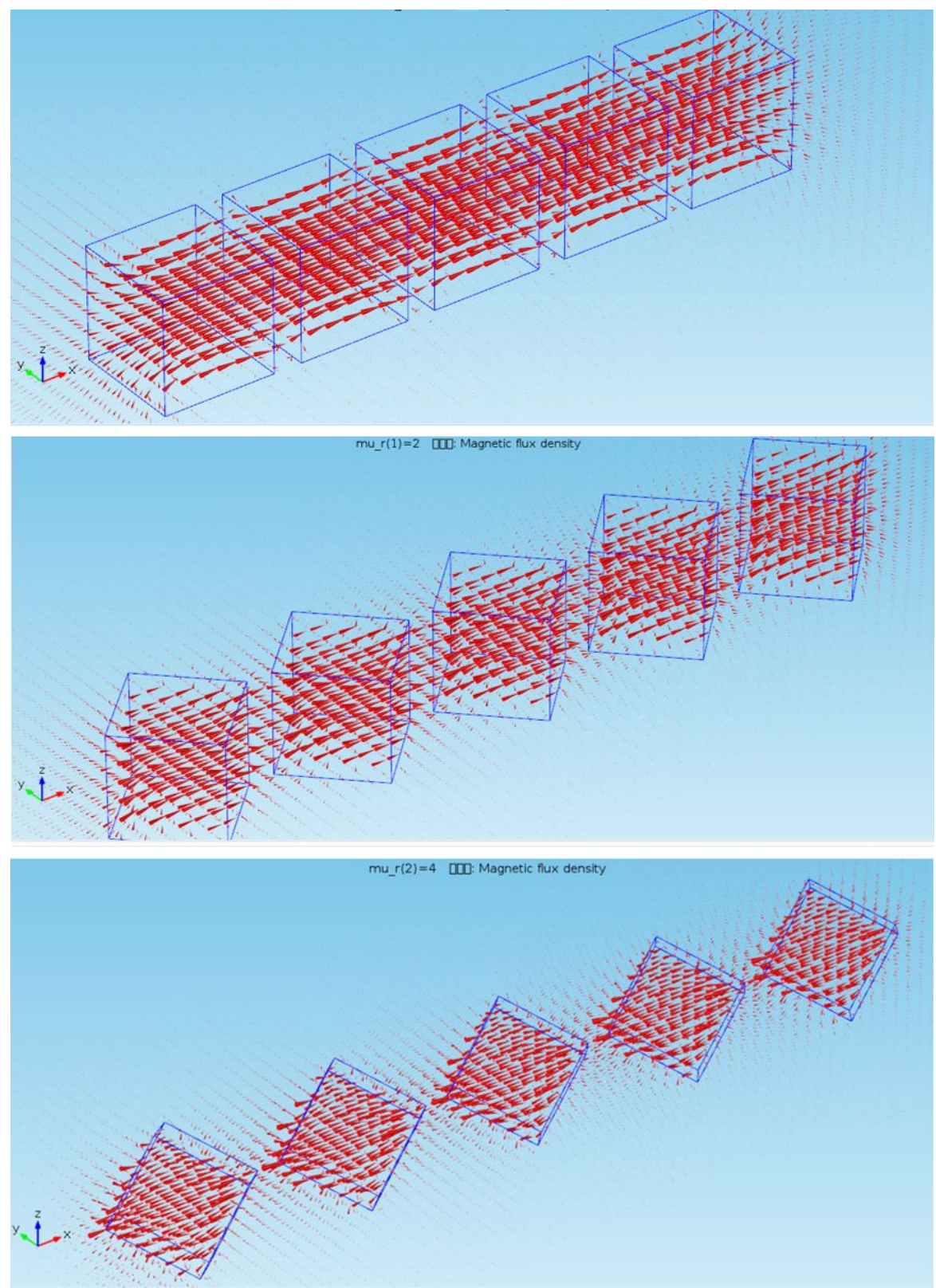

Figure S4. 3D mapping of magnetic flux density in assembled chains along [100], [110] and [111] directions. While the arrows indicate the field direction, their sizes show the magnitude of the field. 


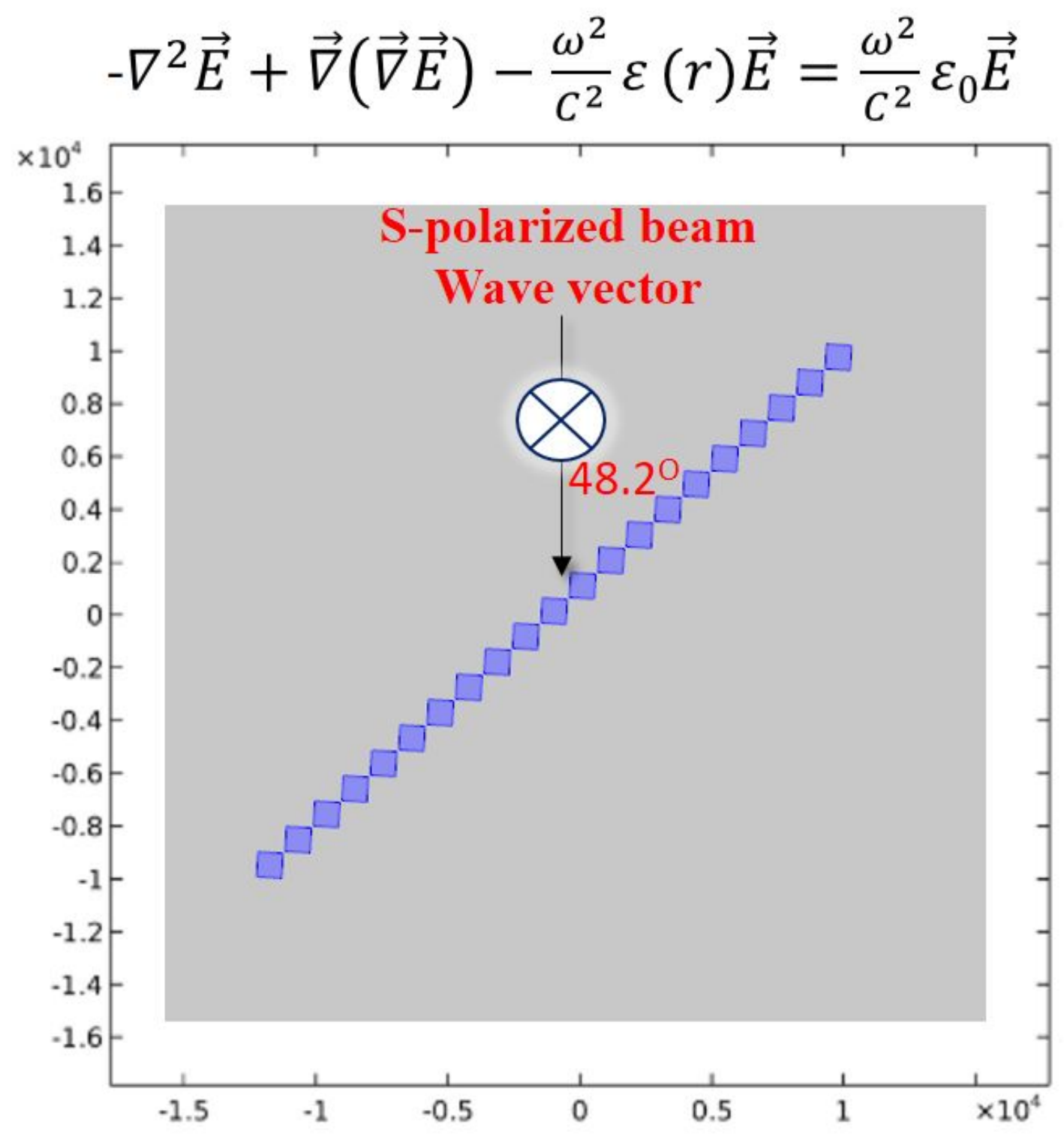

Figure S5. Modeling of light-field splitting by assembled nanochains in wave optics by solving the Maxwell' Equation. 


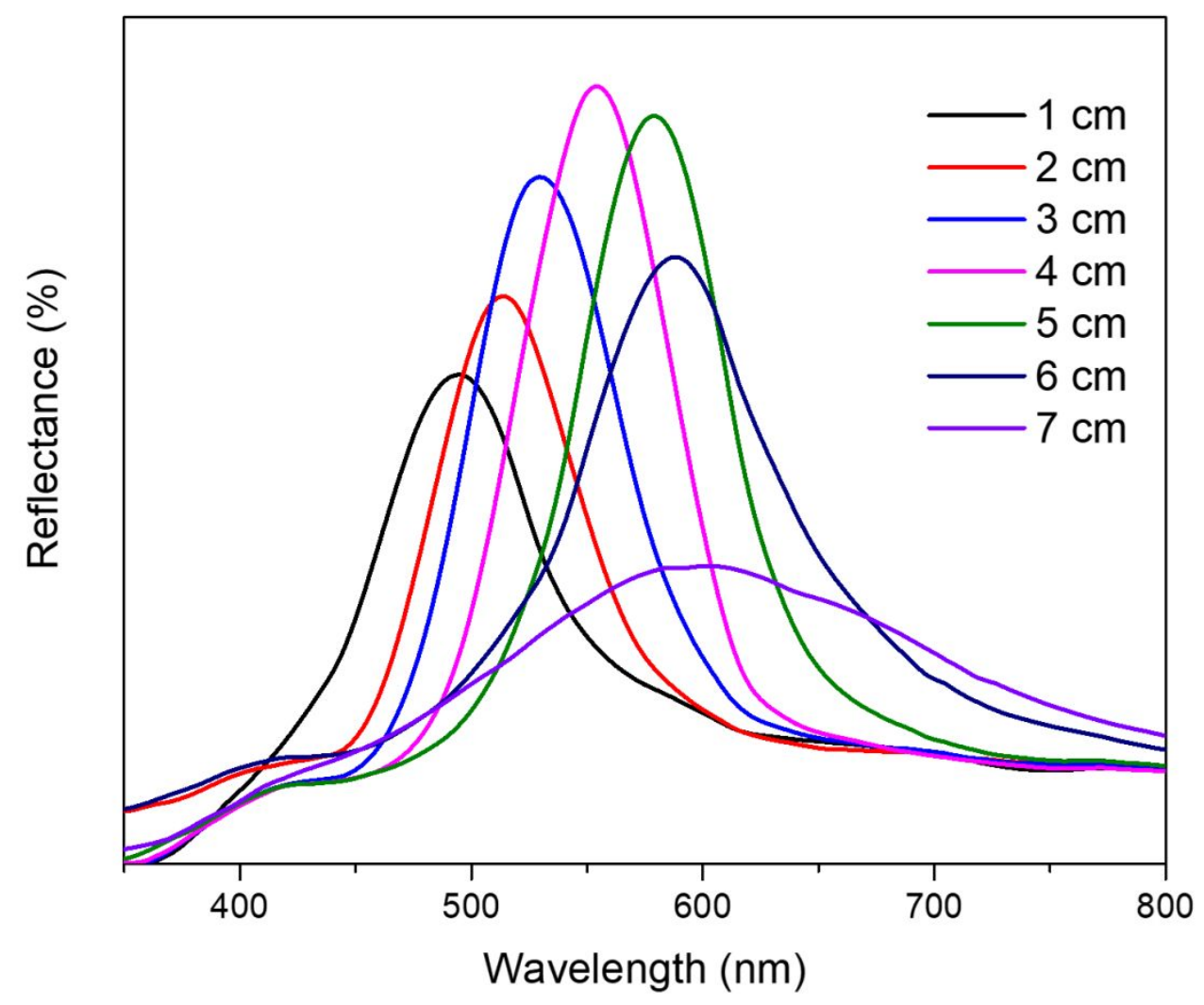

Figure S6. Reflection spectra of an aqueous dispersion of nanocubes under different filed strength by varying the distance between the sample and applied magnet. 
a
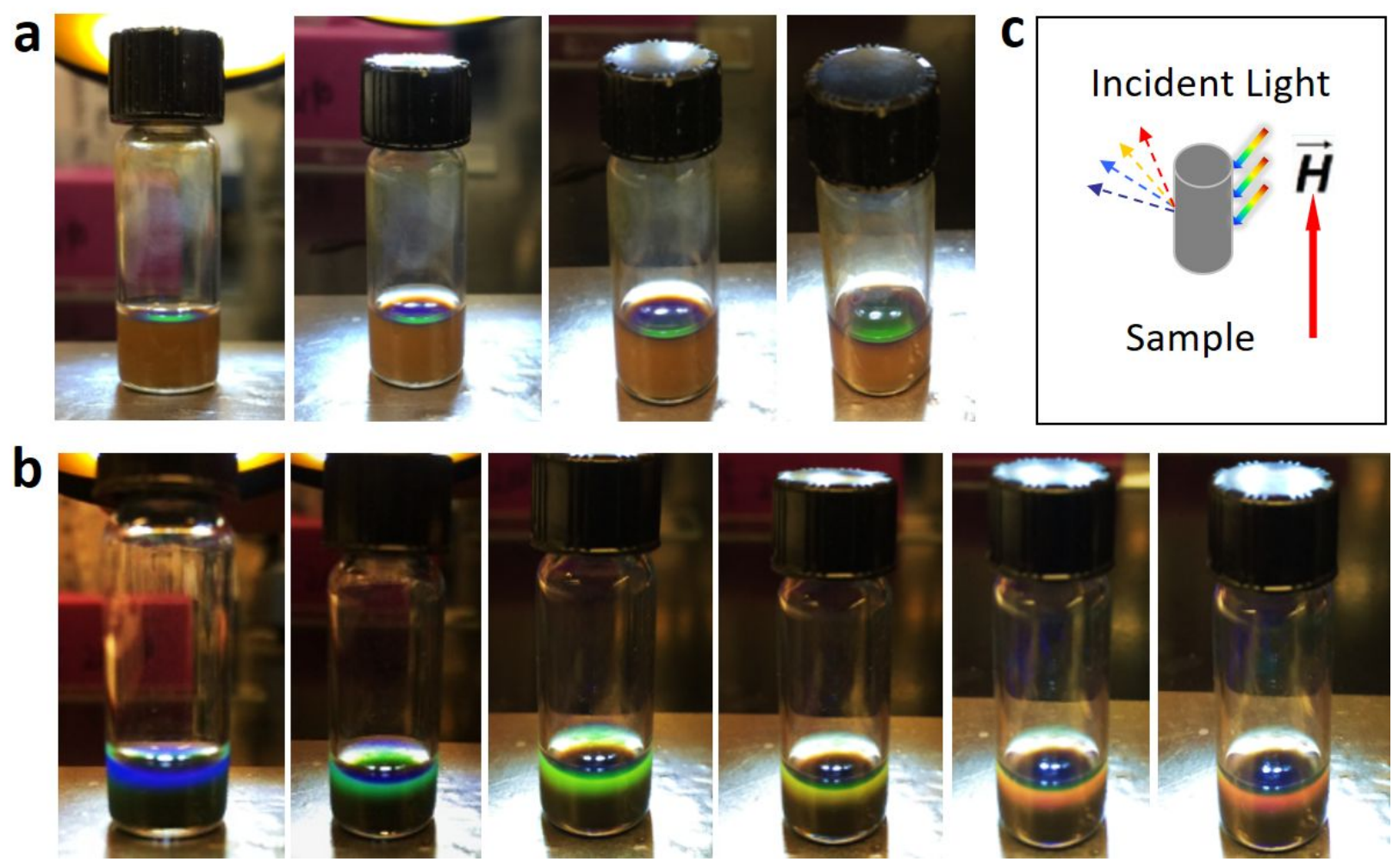

Figure S7. (a) Optical images showing structural color in the colloidal dispersion of CNCs exposed to a vertical magnetic field. (b) Optical images showing structural color in the bulk solution of cubic $\mathrm{Fe}_{3} \mathrm{O}_{4} @ \mathrm{SiO}_{2}$ in glass vail. (c) Scheme showing the diffraction occurring in the colloidal dispersion of cubic $\mathrm{Fe}_{3} \mathrm{O}_{4} @ \mathrm{SiO}_{2}$ exposed to a vertical magnetic field. Images in (a) and (b) were taken at different viewing angles. From left to right, viewing angles decreased gradually. 

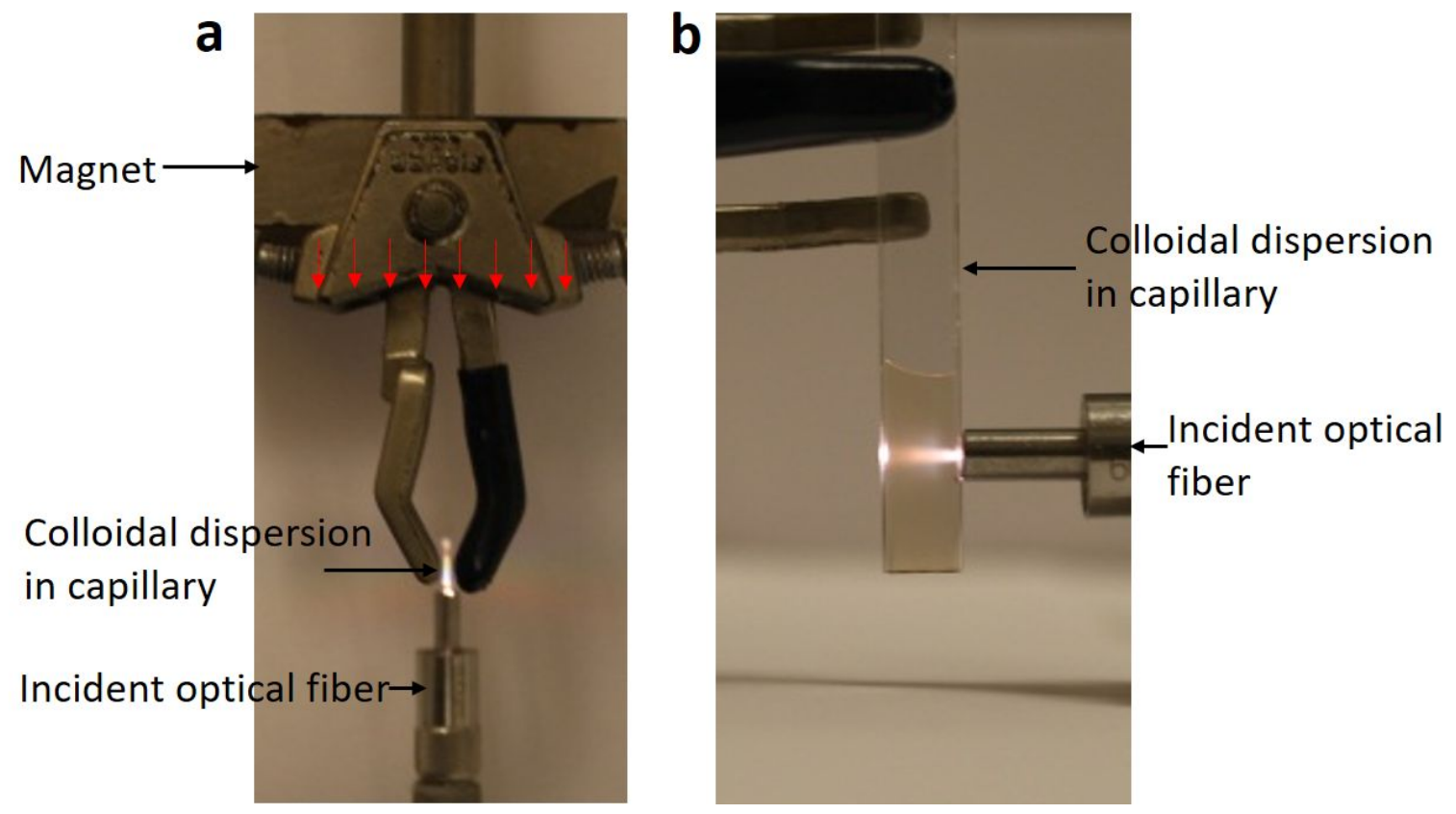

Figure S8. Digital image of setup under (a) top view and (b) side view to visualize the light splitting in Figure 4. Red arrows indicate the field direction. 

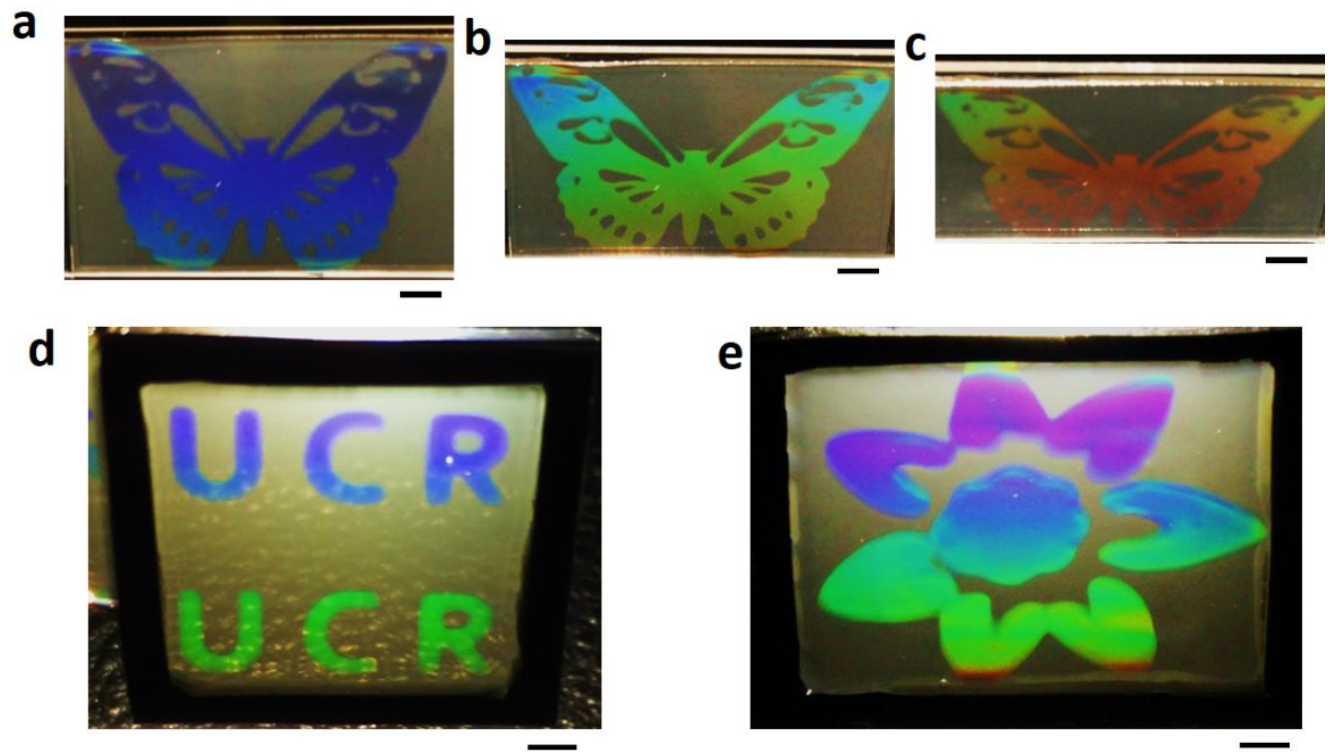

Figure S9. Optical images of fabricated hydrogel films made by fixing chain-structure by lithography with viewing angle decreasing from (a) $60^{\circ}$, (b) $45^{\circ}$ to (c) $30^{\circ}$. (d) and (e) Digital pictures of various printed images. Scale bars: $5 \mathrm{~mm}$ in (a), (b) and (c); $1 \mathrm{~mm}$ in (d) and (e). 


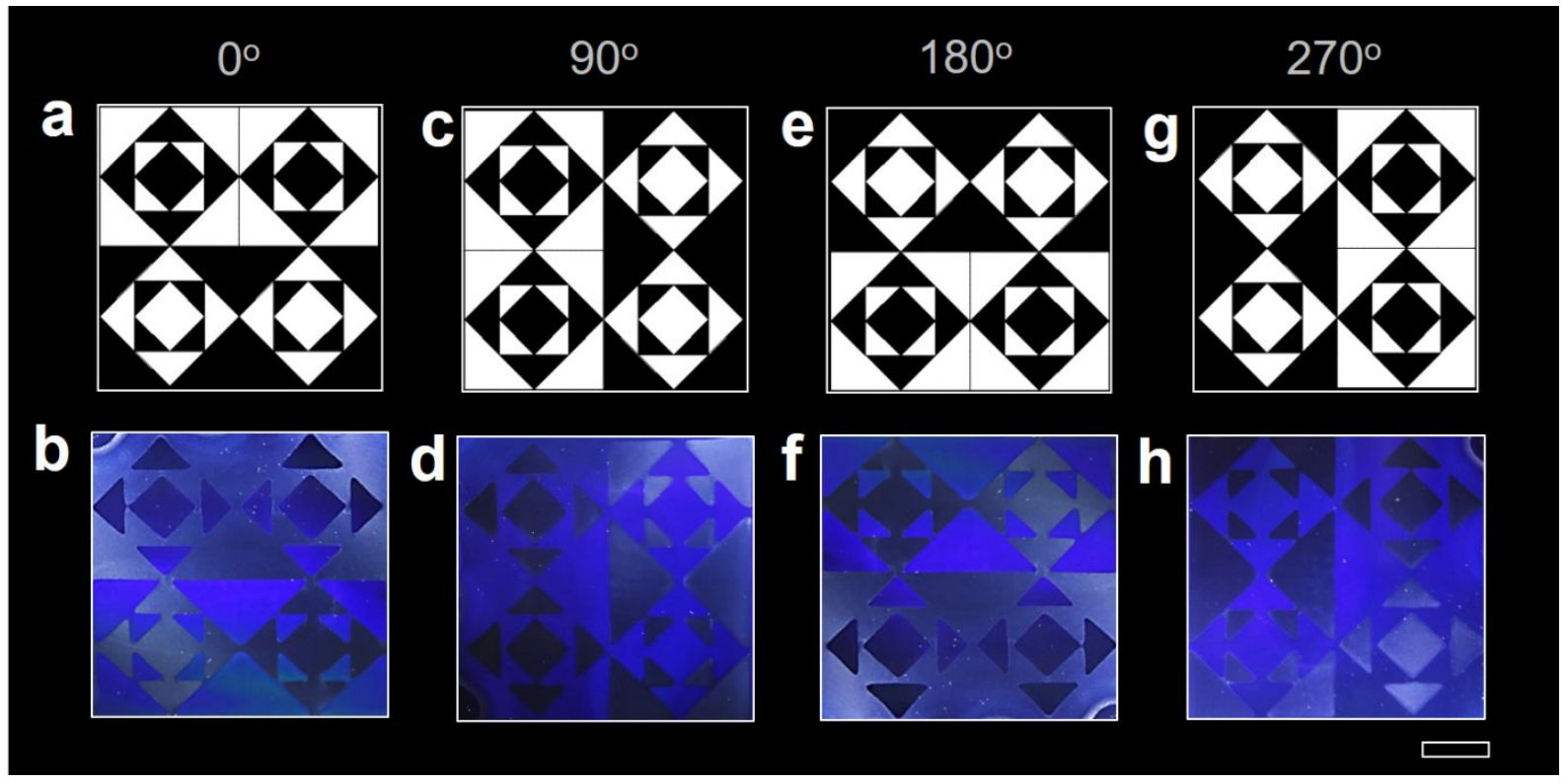

Figure S10. (a), (c), (e) and (g) The counterclockwise rotation of the photomask used for creating the images in photolithography. The switching of the observed patterns follows the same counterclockwise manner. (b), (d), (f) and (h) The created images under the same counterclockwise rotation. Interestingly, the perceived images in the photonic films exhibited an opposite visual effect of clockwise rotation. Scale bar: $5 \mathrm{~mm}$. 